Accepted by The Astronomical Journal

\title{
Radio continuum emission and water masers towards CB 54
}

\author{
Itziar de Gregorio-Monsalvo ${ }^{1}$, José F. Gómez ${ }^{2}$, Guillem Anglada² ${ }^{2}$, José M. Torrelles ${ }^{3}$, \\ Thomas B. H. Kuiper ${ }^{4}$, Olga Suárez ${ }^{2,5}$, Nimesh A. Patel ${ }^{6}$
}

\begin{abstract}
We present high angular resolution observations of water masers at $1.3 \mathrm{~cm}$ and radio continuum emission at 1.3, 3.6 and $6 \mathrm{~cm}$ towards the Bok globule CB 54 using the Very Large Array. At $1.3 \mathrm{~cm}$, with subarsecond angular resolution, we detect a radio continuum compact source located to the south-west of the globule and spatially coincident with a mid-infrared embedded object (MIR-b). The spectral index derived between 6 and $1.3 \mathrm{~cm}(\alpha=0.3 \pm 0.4)$ is flat, consistent with optically thin free-free emission from ionized gas. We propose the shock-ionization scenario as a viable mechanism to produce the radio continuum emission observed at $\mathrm{cm}$ frequencies. Water masers are detected at two different positions separated by $2.3^{\prime \prime}$, and coincide spatially with two mid-infrared sources: MIR-b and MIR-c. The association of these mid-IR sources with water masers confirms that they are likely protostars undergoing mass-loss, and they are the best candidate as driving sources of the molecular outflows in the region.
\end{abstract}

Subject headings: masers — radio continuum:ISM — stars:formation — ISM:globules, jets and outflows, molecules.

\footnotetext{
${ }^{1}$ European Southern Observatory, Alonso de Córdova 3107, Vitacura, Casilla 19001, Santiago 19, Chile

${ }^{2}$ Instituto de Astrofísica de Andalucía (CSIC), Apartado 3004, E-18080 Granada, Spain

${ }^{3}$ Instituto de Ciencias del Espacio (CSIC) and Institut d'Estudis Espacials de Catalunya, C/Gran Capità 2-4, E-08034 Barcelona, Spain.

${ }^{4}$ Jet Propulsion Laboratory, California Institute of Technology, USA

${ }^{5}$ UMR 6525 H. Fizeau, Universit de Nice Sophia Antipolis, CNRS, OCA. Parc Valrose, F-06108 Nice Cedex 2, France

${ }^{6}$ Harvard-Smithsonian Center for Astrophysics, 60 Garden Street, Cambridge, MA 02138, USA
} 


\section{Introduction}

The Bok globule CB 54 is an active star forming region associated with the Vela OB1 cloud complex and located at $1.5 \mathrm{kpc}$ (Launhardt \& Henning 1997). Due to their simplicity and relatively small size $\left(<10^{\prime}\right)$, Bok globules offer an unique environment to study the outcome of the star formation processes in a relatively idealistic and isolated way (Bok \& Reilly 1947; Clemens \& Barvainis 1988).

CB 54 shows different signposts of multiple star formation. The region contains several molecular outflows. There is a main bipolar CO outflow oriented in the northeast-southwest direction and centered near the IRAS point-like source IRAS 07020-1618 (also named CB $54 \mathrm{YC} 1$; Yun \& Clemens 1994b). In addition, several $\mathrm{H}_{2}[v=1-0 \mathrm{~S}(1)]$ line emission knots detected by Khanzadyan (2003) suggest the presence of a second outflow in the east-west direction, which indicates the existence of different driving protostars. In fact, this globule harbors at its center a multiple protostellar system of young stellar objects (YSOs) at different stages of evolution. Near-IR observations towards the central IRAS source revealed the presence of two bright near-IR ( $K$ band, $2.2 \mu \mathrm{m}$ ) objects classified as Class I protostellar candidates, CB 54 YC1-I (a confirmed Class I source; Ciardi \& Gómez-Martín 2007) and CB 54 YC1-II, plus a bright elongated feature (CB 54 YC1-SW) mainly seen in $\mathrm{H}_{2}[v=1$ $0 S(1)$ ], $2.121 \mu \mathrm{m}$ line (Yun \& Clemens 1994a, 1995; Yun et al. 1996; Khanzadvan 2003). Water masers were detected by Gómez et al. (2006) and de Gregorio-Monsalvo et al. (2006) inside this southern elongated feature, suggesting the presence of an embedded protostar that pumps the maser emission. This prediction was recently confirmed by the discovery of three faint mid-IR sources clustered near the position of the IRAS source and within the near-IR elongated feature (Ciardi \& Gómez-Martín 2007). They were named as MIR-a, MIR-b, and MIR-c and interpreted as very cool $(\simeq 100 \mathrm{~K})$ Class 0 protostellar candidates of masses $\sim 1.5 M_{\odot}, \sim 4 M_{\odot}$, and $\sim 0.2 M_{\odot}$ respectively.

Water maser emission at $22 \mathrm{GHz}$ is a good tracer of the mass-loss phenomena observed at the earliest stages of the formation of stars of all masses (Rodríguez et al. 1980; Felli, Palagi, \& Tofani 1992; Xiang \& Turner 1995; De Buizer et al. 2005). In the case of low-mass objects, these water masers are usually associated with the youngest Class 0 protostars, produced by the interaction of powerful jets with a large amount of circumstellar material (Furuya et al. 2001) and they tend to be located close to their powering source (within several hundred AU; Chernin 1995; Claussen et al. 1998; Furuya et al. 2000, 2003). At those earliest stages of evolution young protostars show the most powerful molecular outflows (Bontemps et al. 1996), which are believed to be driven by collimated jets (Raga et al. 1993). The central objects that power the outflows are frequently associated with weak and compact centimeter free-free continuum emission from thermal radio jets (Anglada 1995, 
1996; Beltrán et al. 2001). These radio jets trace the part of the outflow closest to the exciting source. These properties make the combination of water masers and radio continuum emission well suited for pinpointing the location of Class 0 protostars.

In this work we present sensitive interferometric observations of water masers and radio continuum at $1.3 \mathrm{~cm}$, using the Very Large Array (VLA). We also show radio continuum data at 3.6 and $6 \mathrm{~cm}$ from the VLA archive. The main goals of these observations were to derive accurately the position of the water maser emission, to pinpoint the location of the exciting sources of the maser phenomenon, as well as to derive information about the driving engine of the molecular outflows that exist in the region.

This paper is structured as follows: in $\S 2$ we describe the observations and data processing. In $\S 3$ we present and discuss the results derived from radio continuum and water masers observations. Finally, we present the conclusions of this work in $\S 4$.

\section{Observations and data processing}

Observations towards CB 54 were performed on 2005 January 22 and 31, and February 4 using the VLA of the National Radio Astronomy Observatory (NRAO) 1 in the BnA configuration (project AG684). We observed simultaneously the $6_{16}-5_{23}$ transition of $\mathrm{H}_{2} \mathrm{O}$ (rest frequency $=22235.080 \mathrm{MHz}$ ) and continuum at $22285.080 \mathrm{MHz}(\simeq 1.3 \mathrm{~cm})$ using the four IF spectral line mode and processing both right and left circular polarizations. For the $\mathrm{H}_{2} \mathrm{O}$ observations we sampled 64 channels over a bandwidth of $3.125 \mathrm{MHz}$, centered at $V_{\mathrm{LSR}}=15 \mathrm{~km} \mathrm{~s}^{-1}$, with $0.66 \mathrm{~km} \mathrm{~s}^{-1}$ velocity resolution. For continuum observations we used a bandwidth of $25 \mathrm{MHz}$ that comprised 8 channels of $3.125 \mathrm{MHz}$. The total observing time including calibration was 4.5 hours per day. The splitting of the observations into three different days was required to reach the necessary sensitivity for the continuum data. Our flux calibrator was 3C48, for which we adopted a flux density of $1.1 \mathrm{Jy}$ using the latest VLA values (1999.2). The source J0609-157 was used as phase and bandpass calibrator (bootstrapped flux density $=3.90 \pm 0.08 \mathrm{Jy}$ ). The phase center of the observations was R.A.(J2000) $=07^{h} 04^{m} 21^{\mathrm{s}} .4$, Dec(J2000) $=-16^{\circ} 23^{\prime} 15^{\prime \prime}$. The Astronomical Image and Processing System (AIPS), developed by NRAO, was used to calibrate and process our data. We produced $\mathrm{H}_{2} \mathrm{O}$ line maps setting the "robust" weight parameter to 0 , as a compromise between angular resolution and sensitivity. The size of the synthesized beam was $\simeq 0.25^{\prime \prime} \times 0.14^{\prime \prime}$ in the maps of each individual observing day. The water maser emission was strong enough

\footnotetext{
${ }^{1}$ The National Radio Astronomy Observatory is a facility of the National Science Foundation operated under cooperative agreement by Associated Universities, Inc.
} 
to enable self-calibration. Spectral Hanning smoothing was applied to mitigate the Gibbs ringing, which provided a final velocity resolution of $1.3 \mathrm{~km} \mathrm{~s}^{-1}$. The $1.3 \mathrm{~cm}$ continuum data were cross calibrated using the self-calibration solutions obtained from the line data for each individual day and were combined. Continuum maps were obtained using natural weighting to improve the signal to noise ratio, providing a synthesized beam of $\simeq 0.30^{\prime \prime} \times 0.19^{\prime \prime}$ (P.A. $=$ $\left.65^{\circ}\right)$.

We have also reanalyzed radio continuum data at $8.44 \mathrm{GHz}(\simeq 3.6 \mathrm{~cm})$ and $4.86 \mathrm{GHz}$ $(\simeq 6 \mathrm{~cm})$ from the VLA archive (these data were included in the papers by Yun et al. 1996 and Moreira et al. 1997). Both observations were performed with the array in the D configuration for projects AY071 and AY073. A total bandwidth of $100 \mathrm{MHz}$ was selected in the two sets of observations, and both right and left circular polarizations were processed. The time on source was $\simeq 20$ minutes for $8.44 \mathrm{GHz}$ data and $\simeq 1$ hour for $4.86 \mathrm{GHz}$ data. The source $3 \mathrm{C} 48$ was selected as the flux calibrator in both cases (adopted flux density equal to $3.2 \mathrm{Jy}$ and $5.4 \mathrm{Jy}$ respectively for 8.44 and $4.86 \mathrm{GHz}$ frequencies). We have summarized the setup of these archived observations in Table 1.

\section{Results and discussion}

\subsection{Radio continuum emission}

We have detected a compact $\left(\leq 0.2^{\prime \prime}\right)$ continuum source at $1.3 \mathrm{~cm}$ (Table 2) at a position coinciding with the near-infrared elongated feature CB 54 YC1-SW (see Fig. 1). This

feature was proposed to trace an embedded YSO by de Gregorio-Monsalvo et al. (2006) on the basis of its water maser emission, a result that has been recently confirmed by the detection of three mid-infrared sources, within this feature, by Ciardi \& Gómez-Martín (2007), who classified these objects as Class 0 protostellar candidates. Our $1.3 \mathrm{~cm}$ source, is spatially coincident with MIR-b (see Fig. 1), one of the mid-infrared protostars detected by Ciardi \& Gómez-Martín (2007).

Radio continuum emission at 3.6 and $6 \mathrm{~cm}$ is unresolved at both frequencies (see contour maps in Fig. 2). In Table 2 we show detailed information about positions, flux densities, and uncertainties of the continuum emission presented in this section. From this analysis, we see that the position of the radio continuum emission at the three different frequencies is the same within the absolute positional errors, concluding that it comes from the same source, named as CB 54 VLA1 by Yun et al. (1996) and Moreira et al. (1997). 


\subsubsection{Origin of the radio continuum emission}

In order to study the nature of the radio continuum emission associated with CB 54 VLA1, we compare the centimeter continuum luminosity inferred from the radio observations with the centimeter continuum luminosity expected from Lyman-continuum radiation from a ZAMS star of the given luminosity of the source. The bolometric luminosity derived from the flux densities of the source IRAS 07020-1618 close to our radio continuum source is $\sim 344 L_{\odot}$ for an adopted distance of $1.5 \mathrm{kpc}$ (Wang et al. 1995), which corresponds to a B5.5 ZAMS star (Thompson 1984). We warn that the cluster of three YSOs detected by Ciardi \& Gómez-Martín (2007) falls within the positional error ellipsoid of IRAS 07020-1618 and they could contribute to the total luminosity of the IRAS source. Therefore we consider this value as an upper limit. The observed radio continuum luminosity at $1.3 \mathrm{~cm}$ wavelength (i.e., $S_{\nu} d^{2}$ ) is $\sim 7 \times 10^{-1} \mathrm{mJy} \mathrm{kpc}^{2}$. On the other hand, assuming optically thin freefree emission from ionized hydrogen with an electron temperature of $10^{4} \mathrm{~K}$, we derive an expected upper limit of $S_{\nu} d^{2} \lesssim 7 \times 10^{-3} \mathrm{mJy} \mathrm{kpc}^{2}$ from a Lyman-continuum flux of $\sim 7$ $\times 10^{41} \mathrm{~s}^{-1}$ (obtained from Thompson 1984 for a B5.5 ZAMS star). Thus, ionization by stellar photons fails by two orders of magnitude in explaining the observed radio emission, and another ionizing mechanism is required. This behavior has been observed before, for instance, by Torrelles et al. (1985), Rodríguez et al. (1989), and Anglada (1995) for a large set of low-mass YSOs.

A plausible mechanism for explaining the centimeter continuum emission observed is the shock-ionization scenario proposed by Torrelles et al. (1985). In this scenario the stellar wind responsible for a molecular outflow generate shocks in the dense gas surrounding the central protostar and induces its ionization. Curiel et al. (1987, 1989) modeled the shock-ionization scenario and derived the radio continuum emission under optically thin conditions. The spectral index measured by us in the $6-1.3 \mathrm{~cm}$ wavelength range is $\alpha_{6-1.3} \mathrm{~cm}=0.3 \pm 0.4$ (where $\left.S_{\nu} \propto \nu^{\alpha}\right)$, consistent, within the errors, with optically thin free-free emission. The formulation of Curiel et al. (1987, 1989) predicts a correlation between $S_{\nu} d^{2}$ and the momentum rate of the outflow $\dot{P}$. Assuming that the momentum rate in the outflow equals that in the stellar wind, $\dot{P}=\dot{M} v$ (where $\dot{M}$ is the mass loss rate of the wind, and $v$ the terminal velocity of the wind, adopting a typical value of $200 \mathrm{~km} \mathrm{~s}^{-1}$ ), and a typical electron temperature in the ionized wind of $10^{4} \mathrm{~K}$, the prediction of the model gives:

$$
\dot{P}=\frac{10^{-3.5}}{\eta} S_{\nu} d^{2}
$$

with $S_{\nu} d^{2}$ in mJy $\mathrm{kpc}^{2}$ and $\dot{P}$ in $\mathrm{M}_{\odot} \mathrm{yr}^{-1} \mathrm{~km} \mathrm{~s}^{-1}$, being $S_{\nu}$ the flux density at $6 \mathrm{~cm}$ and $\eta=\Omega / 4 \pi$ an efficiency factor that represents the fraction of the stellar wind that is shocked and produces the observed radio continuum emission. Scaling the outflow force derived by 
Yun \& Clemens (1994b) from CO observations, to the adopted distance of $1.5 \mathrm{kpc}$, we obtain $\dot{P}=4 \times 10^{-4} M_{\odot} \mathrm{km} \mathrm{s}^{-1} \mathrm{yr}^{-1}$. Considering a radio continuum luminosity at $6 \mathrm{~cm}$ of 0.5 mJy $\mathrm{kpc}^{2}$, we derive and efficiency factor $\eta \simeq 0.4$, which indicates that the shock-ionization mechanism could explain the observed radio continuum emission. We note that our estimate of efficient factor $\eta$ can be affected by large errors, mainly due to the uncertainty in the value of the momentum rate of the outflow from molecular lines observations (see Anglada et al. 1992 and Anglada 1995 for a detailed discussion of the dependence of the error in $\eta$ with the observational parameters). The efficiency factor derived in this work is somewhat higher than the average value of $\eta \simeq 0.1$ derived by Anglada (1995) for a large set of low luminosity objects. Nevertheless, the dispersion of the efficiency values is relatively large and the best fit to that set of data provides a value of $\eta=10^{-1 \pm 0.6}$ (adopting an uncertainty of $2 \sigma$ ), i.e., $0.025<\eta<0.4$.

The observations presented here make CB 54 VLA1 a very good candidate for driving a molecular outflow. Nevertheless, higher angular resolution observations at centimeter and millimeter wavelengths should be made to study the presence of a jet-disk system, since these structures are typically observed with sizes of $\simeq 100 \mathrm{AU}\left(\simeq 00^{\prime \prime} 07\right.$ at a distance of 1.5

kpc), see Anglada (1996). In particular, high angular resolution observations of 3.6 and $6 \mathrm{~cm}$ emission, optically thicker than that at $1.3 \mathrm{~cm}$, could better trace low-brightness structures, and therefore, would be useful to prove the presence of a thermal radio jet with a morphology elongated in the same direction of the large scale molecular outflow. On the other hand, radio continuum millimeter data would be useful to reveal the presence of heated dust associated with a possible protoplanetary disk.

\subsection{Water maser emission}

Table 3 contains the results of our water masers observations with the VLA. We observe three independent spectral features (see Fig. 3, left panel). One of the features is approximately at the velocity of the cloud $\left(\mathrm{V}_{\mathrm{LSR}}=19.5 \mathrm{~km} \mathrm{~s}^{-1}\right.$; Clemens \& Barvainis 1988) and the rest are blue-shifted within $10 \mathrm{~km} \mathrm{~s}^{-1}$ from the cloud velocity. All of them were detected on the three different days of observation. The masers are found at two different positions separated by $2.286^{\prime \prime} \pm 0.004^{\prime \prime}$ (distance from the southern component to the northern reference feature, $\simeq 3400 \mathrm{AU}$ at a distance of $1.5 \mathrm{kpc}$; see Fig. 11). The northern group of masers is spatially associated with the mid-infrared object MIR-b and shows three spots at velocities 10.4, 13.7, and $19.6 \mathrm{~km} \mathrm{~s}^{-1}$, separated by a few centi-arcseconds (see Fig. 3 , right panel). The southern group is composed by a single spot at a velocity of $9.7 \mathrm{~km} \mathrm{~s}^{-1}$, spatially associated with the mid-infrared object MIR-c. 
The water maser emission in the region shows high variability, which is typically observed in both low and high-mass young stellar objects (Reid \& Moran 1981; Wilking et al. 1994; Claussen et al. 1996). Gómez et al. (2006), using the Robledo $70 \mathrm{~m}$ antenna, detected a water maser spectrum composed of a single water maser spectral feature observed at $V_{\mathrm{LSR}}=$ $13.7 \mathrm{~km} \mathrm{~s}^{-1}$ in 2002 , at $7.9 \mathrm{~km} \mathrm{~s}^{-1}$ in 2003 , and at $8.7 \mathrm{~km} \mathrm{~s}^{-1}$ in 2005 . In addition, de Gregorio-Monsalvo et al. (2006), using the VLA, detected two different features at 15.8 and $17.8 \mathrm{~km} \mathrm{~s}^{-1}$ in February 2004. In the observations reported here, do not detect any of the maser spectral features observed in the mentioned previous works in the region except the feature at $13.7 \mathrm{~km} \mathrm{~s}^{-1}$. This component shows a variation in its intensity by a factor of $\sim 2$ between 2005 January 22 and 2005 January 31 (see Fig. 3, left panel). On the other hand, the features at 10.4 and $19.6 \mathrm{~km} \mathrm{~s}^{-1}$ have not been reported before.

Water masers associated with the northern YSO MIR-b are located at a distance $\leq 100$ $\mathrm{AU}$ (assuming a distance of $1.5 \mathrm{kpc}$ to the Bok globule) from the compact radio continuum source CB 54 VLA1 we detected at $1.3 \mathrm{~cm}$, which suggests this object as the exciting source of the northern group of water masers. This short distance $\leq 100$ AU between water masers and their exciting source is typically observed in a large set of low-mass star forming regions (Chernin 1995; Claussen et al. 1998; Furuya et al. 2000). The right panel of Fig. 3 shows the spatial distribution of the three northern spots obtained on the second day of observation, which corresponds to the data with the best signal to noise ratio. All the spots show similar positions on the three days of observation. They delineate a spatial structure of $\simeq 0.06^{\prime \prime}$ (90 AU), elongated in the north-south direction. To ascertain whether the masers are associated with the molecular outflow or with disk material (i.e., whether they are tracing unbound or bound motions), for each maser component we estimate its velocity $(V)$ with respect to the mean velocity of the maser structure, and we calculate the mass $(M)$ necessary to bind the gas that shows the maser emission as $M=V^{2} R G^{-1}$, where $R$ is the distance of the maser structure to the central YSO, and we have conservatively assumed a value of $R \simeq 100$ AU for the maser components (this distance to the center is an upper limit). A mass of $M \simeq 2.8 M_{\odot}$ is enough to bind the gas responsible for the maser features. Since the mass of this source is estimated to be $\sim 4 M_{\odot}$ (Ciardi \& Gómez-Martín 2007) we cannot discard bound motions.

On the other hand, the spatial association of the southernmost water maser emission with the mid-infrared YSO MIR-c suggests this object as the pumping source of the southern maser emission. In this case, we do not detect $1.3 \mathrm{~cm}$ continuum emission towards this object with a $3 \sigma$ upper limit of $0.17 \mathrm{mJy}$.

Furuya et al. (2001) found that water masers in low-mass YSOs are usually excited by Class 0 sources due to the interaction of powerful jets with a large amount of circumstellar material. Therefore, since sources that host water maser emission are good candidates for 
being in a very early stage of its evolution, as well as for being the exciting source of the mass-loss phenomenon, we propose the sources MIR-b (CB 54 VLA1) and MIR-c as the best candidates to be the driving engine of the molecular outflows that exist in this Bok globule.

\section{Conclusions}

We presented high angular resolution VLA observations of water masers and continuum emission at $1.3 \mathrm{~cm}$ towards the Bok globule CB 54. We complemented our observations with VLA archive data in the radio continuum at 3.6 and $6 \mathrm{~cm}$. The main conclusions are the following:

- Our subarcsecond angular resolution observations at $1.3 \mathrm{~cm}$ allow us to establish that the radio continuum emission detected to the south-west of the Bok globule is associated with the mid-infrared source MIR-b. The spectral index of the emission between 6 and $1.3 \mathrm{~cm}$ is flat, consistent with optically thin free-free emission from ionized gas. A shock scenario mechanism is needed to produce the radio continuum luminosity at cm wavelengths.

- Water masers are found in two different regions. The northern group of masers coincides within $<100 \mathrm{AU}$ with the source CB $54 \mathrm{VLA} 1$, which is associated with the mid-IR protostar MIR-b, and whose position at $1.3 \mathrm{~cm}$ is reported in this paper. The southern region of water maser emission is located $\sim 2.3^{\prime \prime} \mathrm{SW}$, towards the position of the faint mid-IR object MIR-c, without detectable radio continuum emission.

- The association of the mid-IR sources MIR-b and MIR-c with water masers, confirms the embedded protostellar nature of both objects and suggests these protostars are the best candidates for driving the molecular outflows observed in the region.

We thank the referee for providing constructive comments and help in improving the contents of this paper. We are thankful to Per Bergman for his suggestions. GA, IdG, JFG, JMT, and OS are partially supported by Ministerio de Ciencia e Innovación (Spain), grant AYA 2008-06189-C03 (including FEDER funds), and by Consejería de Innovación, Ciencia y Empresa of Junta de Andalucía, (Spain). The work by TBHK was performed in part at the Jet Propulsion Laboratory under contract between the National Aeronautics and Space Administration and the California Institute of Technology.

Facilities: VLA () 


\section{REFERENCES}

Anglada, G. 1995, Revista Mexicana de Astronomía y Astrofísica Conference Series, 1, 67

Anglada, G., Rodríguez, L. F., Canto, J., Estalella, R., \& Torrelles, J. M. 1992, ApJ, 395, 494

Anglada, G. 1996, Radio Emission from the Stars and the Sun, 93, 3

Beltrán, M. T., Estalella, R., Anglada, G., Rodríguez, L. F., \& Torrelles, J. M. 2001, AJ, 121,1556

Bok, B. J., \& Reilly, E. F. 1947, ApJ, 105, 255

Bontemps, S., Andre, P., Terebey, S., \& Cabrit, S. 1996, A\&A, 311, 858

Chernin, L. M. 1995, ApJ, 440, L97

Ciardi, D. R., \& Gómez-Martín, C. 2007, ApJ, 664, 377

Claussen, M. J., Marvel, K. B., Wootten, A., \& Wilking, B. A. 1998, ApJ, 507, L79

Claussen, M. J., Wilking, B. A., Benson, P. J., Wootten, A., Myers, P. C., \& Terebey, S. 1996, ApJS, 106, 111

Clemens, D. P. \& Barvainis, R. 1988, ApJS, 68, 257

Curiel, S., Canto, J., \& Rodríguez, L. F. 1987, Revista Mexicana de Astronomía y Astrofísica, vol. 14, 14, 595

Curiel, S., Rodríguez, L. F., Bohigas, J., Roth, M., Cantó, J., \& Torrelles, J. M. 1989, Astrophysical Letters Communications, 27, 299

De Buizer, J. M., Radomski, J. T., Telesco, C. M., \& Piña, R. K. 2005, ApJS, 156, 179

de Gregorio-Monsalvo, I., Gómez, J. F., Suárez, O., Kuiper, T. B. H., Anglada, G., Patel, N. A., \& Torrelles, J. M. 2006, AJ, 132, 2584

Felli, M., Palagi, F., \& Tofani, G. 1992, A\&A, 255, 293

Furuya, R. S., Kitamura, Y., Wootten, H. A., Claussen, M. J., Saito, M., Marvel, K. B., \& Kawabe, R. 2000, ApJ, 542, L135

Furuya, R. S., Kitamura, Y., Wootten, H. A., Claussen, M. J., \& Kawabe, R. 2001, ApJ, 559, L143 
Furuya, R. S., Kitamura, Y., Wootten, A., Claussen, M. J., \& Kawabe, R. 2003, ApJS, 144, 71

Gómez, J. F., de Gregorio-Monsalvo, I., Suárez, O., \& Kuiper, T. B. H. 2006, AJ, 132, 1322

Khanzadyan, T. 2003, Ph.D. Thesis

Launhardt, R. \& Henning, T. 1997, A\&A, 326, 329

Moreira, M. C., Yun, J. L., Vázquez, R., \& Torrelles, J. M. 1997, AJ, 113, 1371.

Raga, A. C., Canto, J., Calvet, N., Rodríguez, L. F., \& Torrelles, J. M. 1993, A\&A, 276, 539

Reid, M. J. \& Moran, J. M. 1981, ARA\&A, 19, 231

Reynolds, S. P. 1986, ApJ, 304, 713

Rodríguez, L. F. 2000 in Disks, Planetesimals, and Planets, ASP Conf. Ser., 219, 108

Rodríguez, L. F., Myers, P. C., Cruz-Gonzalez, I., \& Terebey, S. 1989, ApJ, 347, 461

Rodríguez, L. F., Moran, J. M., Gottlieb, E. W., \& Ho, P. T. P. 1980, ApJ, 235, 845

Rodríguez, L. F., D’Alessio, P., Wilner, D. J., Ho, P. T P., Torrelles, J. M., Curiel, S., Gomez, Y., Lizano, S., Pedlar, A., Canto, J., \& Raga, A. C. 1998, Nature, 395, 355

Thompson, R. I. 1984, ApJ, 283, 165

Torrelles, J. M., Ho, P. T. P., Rodríguez, L. F., \& Cantó, J. 1985, ApJ, 288, 595

Wang, Y., Evans, N. J., Zhou, S., \& Clemens, D. P. 1995, ApJ, 454, 217

Wilking, B. A., Claussen, M. J., Benson, P. J., Myers, P. C., Terebey, S., \& Wootten, A. 1994, ApJ, 431, L119

Xiang, D. \& Turner, B. E. 1995, ApJS, 99, 121

Yun, J. L. \& Clemens, D. P. 1994a, AJ, 108, 612

Yun, J. L. \& Clemens, D. P. 1994b, ApJS, 92, 145

Yun, J. L. \& Clemens, D. P. 1995, AJ, 109, 742

Yun, J. L., Moreira, M. C.,Torrelles, J. M., Afonso, J. M., \& Santos, N. C. 1996, AJ, 111, 841 
Yun, J. L. 1996, AJ, 111, 930

This preprint was prepared with the AAS LATEX macros v5.2. 


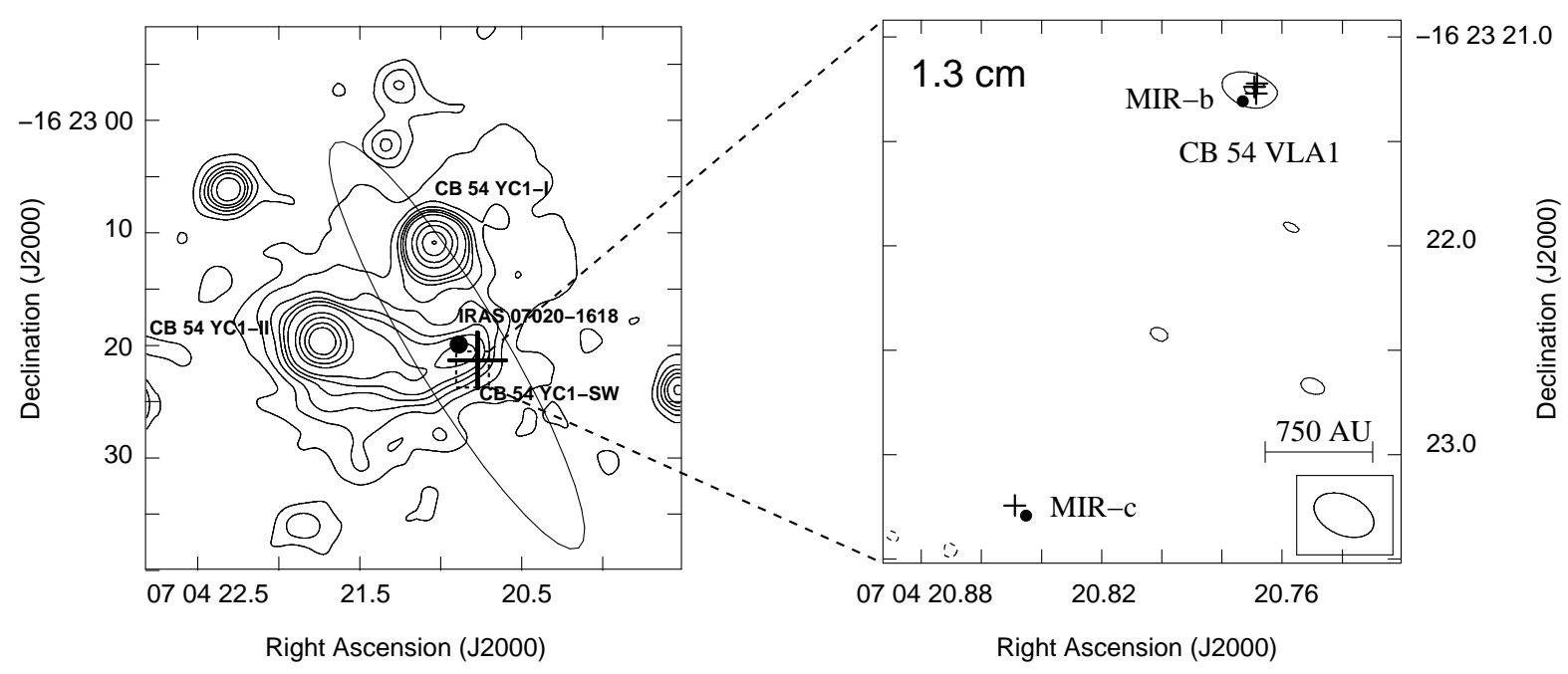

Fig. 1.- Left: Contour plot of the 2MASS near-IR emission towards CB 54, adapted from de Gregorio-Monsalvo et al. (2006). The ellipse represents the positional error of IRAS 07020-1618, whose nominal position is marked with a filled circle. The cross represents the water maser emission detected by de Gregorio-Monsalvo et al. (2006). Right: Contour map of the $1.3 \mathrm{~cm}$ continuum emission observed with the BnA configuration of the VLA. Levels are $-3,3$, and 5 times $0.06 \mathrm{mJy} \mathrm{beam}^{-1}$, the rms of the map. The HPBW of the synthesized beam is shown in the lower-right corner. Crosses mark the positions of the two groups of water masers detected in this work and observed simultaneously with the 1.3 $\mathrm{cm}$ continuum emission. Filled circles represent the mid-infrared sources MIR-b and MIR-c detected by Ciardi \& Gómez-Martín (2007). 

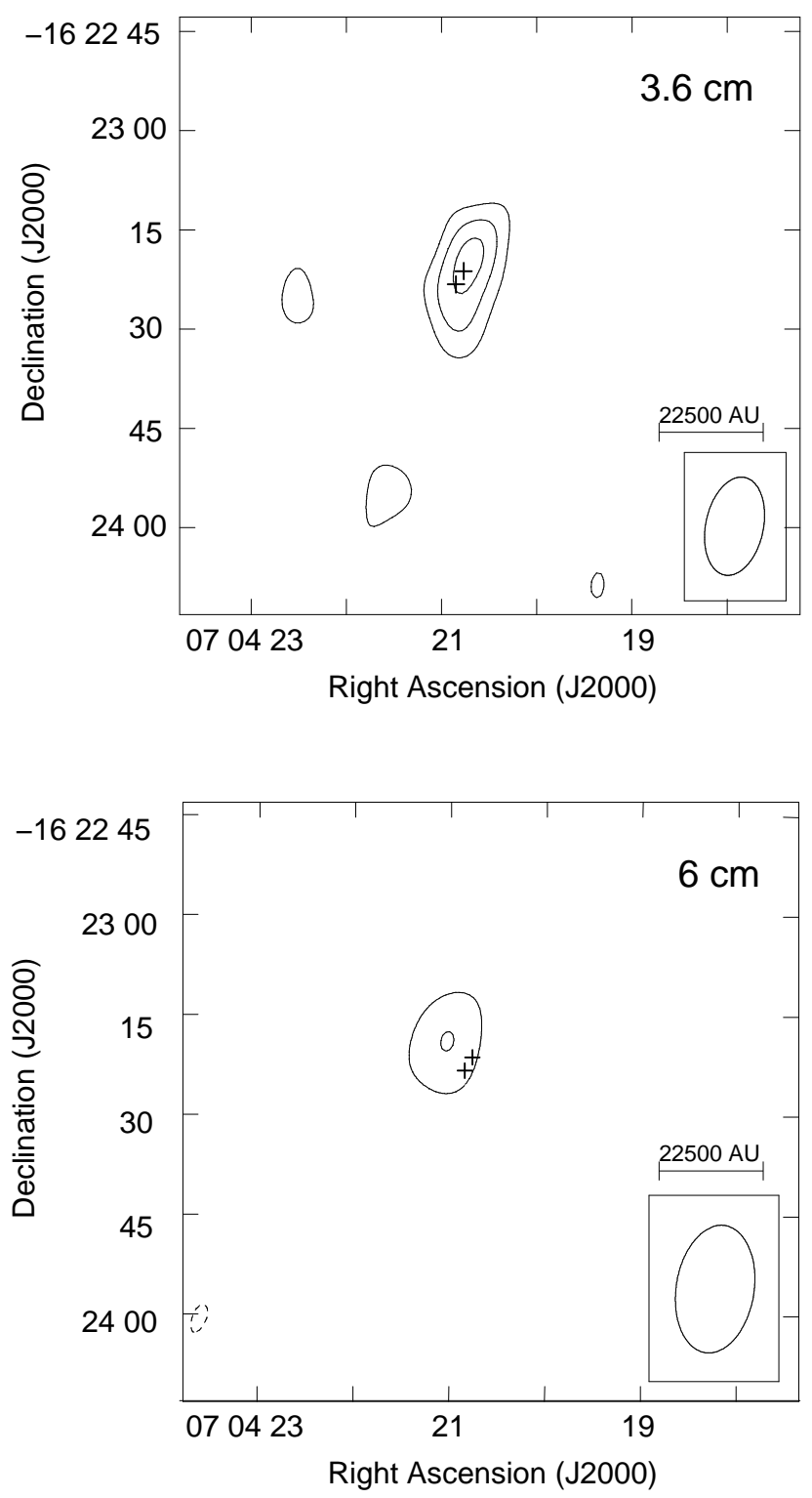

Fig. 2.- Map of radio continuum emission observed at 3.6 and $6 \mathrm{~cm}$. Contour levels are -3 , 3,5 and 7 times the rms of the map $\left(0.025 \mathrm{mJy} \mathrm{beam}^{-1}\right)$ for data at $3.6 \mathrm{~cm}$ wavelength, and $-3,3$ and 5 times the rms of the map $\left(0.045 \mathrm{mJy}_{\text {beam }}^{-1}\right)$ for data at $6 \mathrm{~cm}$. Crosses mark the positions of the two groups of water masers detected in this work. The HPBW of the synthesized beam is represented in the lower-right corner of each plot. 

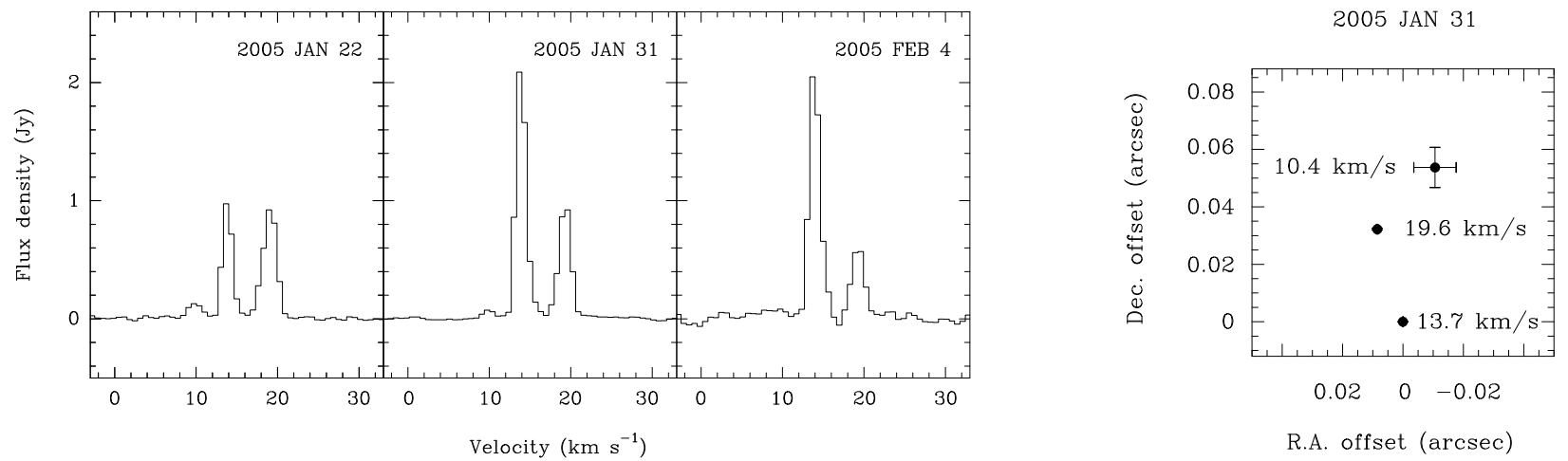

Fig. 3.- Left: Spectra of the water maser emission observed with the VLA on 2005 January 22 (left), January 31 (center), and February 4 (right), integrated over a region of $2.1^{\prime \prime} \times 2.7^{\prime \prime}$, which includes the emission from the two groups of masers that are associated with MIR-b (CB54 VLA1) and MIR-c. Right: Positions of the independent water maser spectral features of the northern group of masers, which are associated with MIR-b (CB54 VLA1), as shown in the $1.3 \mathrm{~cm}$ map of Fig. 1. The positions correspond to the 2005 January 31 observation. The $(0,0)$ is the position of the reference feature used for self-calibration, and the size of the crosses represent the relative position uncertainty with respect to the reference feature (see Table 1). The southern group of water masers show one spot at $9.7 \mathrm{~km} \mathrm{~s}^{-1}$. 
Table 1. Setup of VLA archive data analyzed

\begin{tabular}{ccccccccc}
\hline \hline $\begin{array}{c}\text { Freq. } \\
(\mathrm{GHz})\end{array}$ & $\begin{array}{c}\text { Observation } \\
\text { date }\end{array}$ & $\begin{array}{c}\text { Project } \\
\text { name }\end{array}$ & $\begin{array}{c}\text { R.A. }^{\mathrm{a}} \\
(\mathrm{J} 2000)\end{array}$ & $\begin{array}{c}\text { Dec. } \\
(\mathrm{J} 2000)\end{array}$ & $\begin{array}{c}\text { Beam Size } \\
\left({ }^{\prime \prime} \times{ }^{\prime \prime}\right)\end{array}$ & P.A. & $\begin{array}{c}\text { Phase } \\
\text { calibrator }\end{array}$ & $\begin{array}{c}S_{\text {cal }}{ }^{\mathrm{b}} \\
(\mathrm{Jy})\end{array}$ \\
\hline 8.44 & 19-MAY-1995 & AY071 & $07^{h} 04^{m} 20.9$ & $-16^{\circ} 23^{\prime} 20^{\prime \prime}$ & $15 \times 9$ & $-12^{\circ}$ & J0609-157 & $5.60 \pm 0.01$ \\
4.86 & 30-JUN-1996 & AY073 & $07^{h} 04^{m} 21^{\mathrm{s}} 2$ & $-16^{\circ} 23^{\prime} 15^{\prime \prime}$ & $19 \times 12$ & $-9^{\circ}$ & J0729-366 & $2.845 \pm 0.006$ \\
\hline
\end{tabular}

${ }^{\text {a }}$ Coordinates of the phase center.

${ }^{\mathrm{b}}$ Bootstrapped flux densities of phase calibrators. 
Table 2. Radio continuum emission towards CB 54

\begin{tabular}{cllcc}
\hline \hline $\begin{array}{c}\text { Frequency } \\
(\mathrm{GHz})\end{array}$ & \multicolumn{1}{c}{$\begin{array}{c}\text { R.A. } \\
(\mathrm{J} 2000)\end{array}$} & $\begin{array}{c}\text { Dec. } \\
(\mathrm{J} 2000)\end{array}$ & $\begin{array}{c}\text { Position }^{\mathrm{a}} \\
\text { uncertainty }\left({ }^{\prime \prime}\right)\end{array}$ & $\begin{array}{c}\text { Flux density } \\
(\mathrm{mJy})\end{array}$ \\
\hline 22.24 & $07^{h} 04^{m} 20^{\mathrm{s}} .771$ & $-16^{\circ} 23^{\prime} 21^{\prime \prime} .26$ & 0.08 & $0.29 \pm 0.11$ \\
8.44 & $07^{h} 04^{m} 20.73$ & $-16^{\circ} 23^{\prime} 21^{\prime \prime} .7$ & 2.3 & $0.19 \pm 0.05$ \\
4.86 & $07^{h} 04^{m} 21^{\mathrm{s}} .0$ & $-16^{\circ} 23^{\prime} 19^{\prime \prime}$ & 4 & $0.23 \pm 0.09$ \\
\hline
\end{tabular}

Note. - Uncertainties are $2 \sigma$.

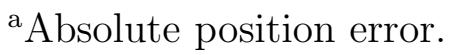


Table 3. Water Maser Features Detected with the VLA towards CB 54

\begin{tabular}{ccccrc}
\hline \hline Date & $\begin{array}{c}\text { R.A. Offset } \\
\left({ }^{\mathrm{a}}\right)\end{array}$ & $\begin{array}{c}\text { Dec.Offset } \\
\left({ }^{\prime \prime}\right)\end{array}$ & $\begin{array}{c}\text { Position }^{\mathrm{b}} \\
\text { Uncertainty }^{\left({ }^{\prime \prime}\right)}\end{array}$ & $\begin{array}{c}\text { Flux Density } \\
(\mathrm{mJy})\end{array}$ & $\begin{array}{c}V_{\text {LSR }}{ }^{\mathrm{c}} \\
\left(\mathrm{km} \mathrm{s}^{-1}\right)\end{array}$ \\
\hline \multirow{2}{*}{ 2005 JAN 22 } & 0.0094 & 0.0318 & 0.0004 & $913 \pm 3$ & 19.6 \\
& 0 & 0 & $\ldots . \mathrm{d}$ & $928 \pm 3$ & 13.7 \\
& -0.011 & 0.046 & 0.012 & $31 \pm 3$ & 10.4 \\
& 1.152 & -1.972 & 0.004 & $106 \pm 3$ & 9.7 \\
\hline \multirow{2}{*}{ 2005 JAN 31 } & 0.00848 & 0.03220 & 0.00027 & $913 \pm 2$ & 19.6 \\
& 0 & 0 & $\ldots . \mathrm{d}$ & $2073 \pm 2$ & 13.7 \\
& -0.011 & 0.057 & 0.007 & $35 \pm 2$ & 10.4 \\
& 1.152 & -1.974 & 0.003 & $74 \pm 2$ & 9.7 \\
\hline & 0.0093 & 0.0328 & 0.0013 & & \\
& 0 & 0 & $\ldots . \mathrm{d}$ & $2080 \pm 6$ & 13.7 \\
& -0.014 & 0.056 & 0.023 & $33 \pm 6$ & 10.4 \\
& 1.149 & -1.971 & 0.013 & $57 \pm 6$ & 9.7 \\
\hline
\end{tabular}

Note. - Quoted uncertainties in this table are $2 \sigma$

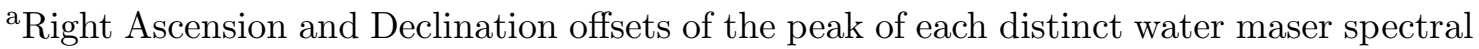
feature with respect to the reference feature used for self-calibration, whose position is R.A.(J2000.0 $)=07^{h} 04^{m} 20^{\mathrm{s}} .769$, Dec. $(\mathrm{J} 2000.0)=-16^{\circ} 23^{\prime} 21^{\prime \prime} .27$, in the three observation days. The absolute position uncertainty of this reference position is $\simeq 0^{\prime \prime} 05$.

${ }^{\mathrm{b}}$ Uncertainty in the relative positions with respect to the reference position.

${ }^{\mathrm{c}} \mathrm{LSR}$ velocity of the spectral features. Velocity resolution is $\sim 1.3 \mathrm{~km} \mathrm{~s}^{-1}$.

${ }^{\mathrm{d}}$ Reference feature. 